Bull. Korean Math. Soc. 49 (2012), No. 2, pp. 295-306

http://dx.doi.org/10.4134/BKMS.2012.49.2.295

\title{
MAXIMALITY PRESERVING CONSTRUCTIONS OF MAXIMAL COMMUTATIVE SUBALGEBRAS OF MATRIX ALGEBRA
}

\author{
YOUNGKWON SONG
}

\begin{abstract}
Let $\left(R, m_{R}, k\right)$ be a local maximal commutative subalgebra of $M_{n}(k)$ with nilpotent maximal ideal $m_{R}$. In this paper, we will construct a maximal commutative subalgebra $R^{S T}$ which is isomorphic to $R$ and study some interesting properties related to $R^{S T}$. Moreover, we will introduce a method to construct an algebra in $M C_{n}(k)$ with $i\left(m_{R}\right)=n$ and $\operatorname{dim}(R)=n$.
\end{abstract}

\section{Introduction}

Throughout this paper, $\left(R, m_{R}, k\right)$ is a local maximal commutative subalgebra of $M_{n}(k)$ with nilpotent maximal ideal $m_{R}$ and residue class field $k$. The set of all local maximal commutative subalgebras $\left(R, m_{R}, k\right)$ of $M_{n}(k)$ will be denoted by $M C_{n}(k)$. The socle of the algebra $R$ is denoted by $\operatorname{soc}(R)$ and $i\left(m_{R}\right)$ is the index of nilpotency of the maximal ideal $m_{R}$. Also, we will let $E_{i j}$ be the $(i, j)$-th matrix unit.

The next theorem is known as the Kravchuk's theorem [10].

Theorem $1.1([6],[10])$. Let $\left(R, m_{R}, k\right)$ be an algebra in $M C_{n}(k)$ with $i\left(m_{R}\right) \geq$ 3. Then, the matrix $r$ in $m_{R}$ can be assumed to be of the following form:

$$
r=\left(\begin{array}{lll}
O_{\ell \times \ell} & O_{\ell \times p} & O_{\ell \times q} \\
A(r)_{p \times \ell} & B(r)_{p \times p} & O_{p \times q} \\
C(r)_{q \times \ell} & D(r)_{q \times p} & O_{q \times q}
\end{array}\right) .
$$

Here, $n=\ell+p+q, \ell \neq 0, p \neq 0, q \neq 0$. Moreover, $\operatorname{soc}(R)$ consists of all matrices of the form:

$$
r=\left(\begin{array}{ll}
O_{(n-q) \times \ell} & O_{(n-q) \times(n-\ell)} \\
C(r)_{q \times \ell} & O_{q \times(n-\ell)}
\end{array}\right) .
$$

Received October 29, 2010.

2010 Mathematics Subject Classification. 15A27, 15A33.

Key words and phrases. maximal commutative subalgebra, ST-isomorphism.

The present research has been supported by the Research Grant of Kwangwoon University in 2011 . 
Remark $1.2([6],[10])$. Let $\left(R, m_{R}, k\right)$ be an algebra in $M C_{n}(k)$. If $i\left(m_{R}\right)=3$, then the matrix $r$ in $m_{R}$ can be assumed to be of the following form:

$$
r=\left(\begin{array}{lll}
O_{\ell \times \ell} & O_{\ell \times p} & O_{\ell \times q} \\
A(r)_{p \times \ell} & O_{p \times p} & O_{p \times q} \\
C(r)_{q \times \ell} & D(r)_{q \times p} & O_{q \times q}
\end{array}\right),
$$

where $n=\ell+p+q, \ell \neq 0, p \neq 0, q \neq 0$.

Theorem $1.3([10])$. Let $\left(R, m_{R}, k\right)$ be an algebra in $M C_{n}(k)$. Suppose the matrices $r_{i}$ in $m_{R}$ of the form

$$
r_{i}=\left(\begin{array}{lll}
O_{\ell \times \ell} & O_{\ell \times p} & O_{\ell \times q} \\
A\left(r_{i}\right)_{p \times \ell} & B\left(r_{i}\right)_{p \times p} & O_{p \times q} \\
C\left(r_{i}\right)_{q \times \ell} & D\left(r_{i}\right)_{q \times p} & O_{q \times q}
\end{array}\right), \quad i=1,2, \ldots, t
$$

constitute a basis for $m_{R}$. Then, the rank of the following $p \times \ell t$ matrix $H$ is $p$ :

$$
H=\left(\begin{array}{llll}
A\left(r_{1}\right) & A\left(r_{2}\right) & \cdots & A\left(r_{t}\right)
\end{array}\right) .
$$

In Section 2, we will construct a maximal commutative subalgebra $R^{S T}$ which is isomorphic to $R$ and study some interesting properties related to $R^{S T}$.

In Section 3, we will find some conditions that the algebra $S=\left\{r \in R \mid r^{S T}=\right.$ $r$ \} can be an algebra in $M C_{n}(k)$.

In Section 4, we will introduce a method to construct an algebra in $M C_{n}(k)$ with $i\left(m_{R}\right)=n$ and $\operatorname{dim}(R)=n$ such that $r=r^{S T}$ for all $r \in R$.

\section{ST-isomorphism}

In this section, we will first define the skew transpose matrix $r^{S T}$ of a matrix $r$ in $M_{m \times n}(k)$ and construct some interesting isomorphic algebras $R^{S T}$ in $M C_{n}(k)$.

Definition 2.1. Let $A=\left(a_{i j}\right)_{m \times n} \in M_{m \times n}(k)$ be a matrix with $a_{i j}$ as its $(i, j)$-th entry. Define $A^{S T}=\left(b_{i j}\right)_{n \times m} \in M_{n \times m}(k)$ be the matrix as follows:

$$
b_{i j}=a_{(m-j+1)(n-i+1)} \quad(1 \leq i \leq n, 1 \leq j \leq m) .
$$

We will call the matrix $A^{S T}$ the skew transpose of $A$.

Thus, the skew transpose $A^{S T}$ of $A$ is the following $n \times m$ matrix:

$$
A^{S T}=\left(\begin{array}{lll}
a_{m n} & \cdots & a_{1 n} \\
\vdots & \ddots & \vdots \\
a_{m 1} & \cdots & a_{11}
\end{array}\right) .
$$

Let $A=\left(a_{i j}\right)_{n \times n} \in M_{n}(k)$ be a square matrix. If we call the line from $(1, n)$ th entry to $(n, 1)$-th entry as the skew-diagonal line, then the skew transpose $A^{S T}$ can be obtained by symmetric moving the entries of $A$ with respect to the skew-diagonal line of $A$.

For the skew transpose $A^{S T}$ of the matrix $A$, the following properties can be easily proved. 
Theorem 2.2. Let $A, B \in M_{m \times n}(k)$ and $\alpha \in k$. Then the following properties hold:

(1) $(A+B)^{S T}=A^{S T}+B^{S T}$,

(2) $(\alpha A)^{S T}=\alpha A^{S T}$,

(3) $\left(A^{S T}\right)^{S T}=A$,

(4) $\left(A^{T}\right)^{S T}=\left(A^{S T}\right)^{T}$, where $A^{T}$ is the transpose of $A$.

Theorem 2.3. Let $A \in M_{m \times n}(k)$ and $B \in M_{n \times \ell}(k)$. Then $(A B)^{S T}=$ $B^{S T} A^{S T}$

Theorem 2.4. Suppose $R$ is an algebra in $M C_{n}(k)$. Then the algebra $R^{S T}=$ $\left\{r^{S T} \mid r \in R\right\}$ is also in $M C_{n}(k)$.

Proof. Define a map $f: R \rightarrow R^{S T}$ by $f(r)=r^{S T}$ for all $r \in R$. Then, straightforward computations show that $f$ is an isomorphism as $k$-algebras by the properties in Theorem 2.2 and Theorem 2.3. Thus, the algebra $R^{S T}$ is also in $M C_{n}(k)$.

Definition 2.5. The isomorphism $f$ in the proof of Theorem 2.4 will be called an ST-isomorphism as $k$-algebras.

By Theorem 2.4, the ST-isomorphism as $k$-algebras is a maximality preserving map.

Example 2.6. (1) Let $R$ be the following algebra in $M C_{4}(k)$ :

$$
R=\left\{\left(\begin{array}{cccc}
a & 0 & 0 & 0 \\
b & a & 0 & 0 \\
c & b & a & 0 \\
d & 0 & 0 & a
\end{array}\right) \mid a, b, c, d \in k\right\} .
$$

Then,

$$
R^{S T}=\left\{\left(\begin{array}{cccc}
a & 0 & 0 & 0 \\
0 & a & 0 & 0 \\
0 & b & a & 0 \\
d & c & b & a
\end{array}\right) \mid a, b, c, d \in k\right\}
$$

is also an algebra in $M C_{4}(k)$.

(2) Let $R$ be the following algebra in $M C_{4}(k)$ :

$$
R=\left\{\left(\begin{array}{cccc}
a & 0 & 0 & 0 \\
b & a & 0 & 0 \\
c & b & a & 0 \\
d & c & b & a
\end{array}\right) \mid a, b, c, d \in k\right\} .
$$

Then, $R^{S T}=R$ and is also an algebra in $M C_{4}(k)$.

More generally, if we let $R=k\left[E_{21}+\cdots+E_{n n-1}, E_{31}+\cdots+E_{n n-2}, \ldots, E_{n 1}\right]$ be an algebra in $M C_{n}(k)$. Then, $R^{S T}=R$ and is also an algebra in $M C_{n}(k)$.

Using the properties of skew transpose matrix, the following properties can be proved: 
Theorem 2.7. Suppose $R$ is an algebra in $M C_{n}(k)$. If $f: R \rightarrow R^{S T}$ is the $S T$-isomorphism as $k$-algebras, then the following properties hold:

(1) $\alpha f: R \longrightarrow R^{S T}$ defined by $(\alpha f)(r)=\alpha f(r)$ for all $r \in R$ is a maximality preserving isomorphism as $k$-vector spaces for all nonzero $\alpha$ in $k$.

(2) $f^{-1}: R^{S T} \longrightarrow R$ is a maximality preserving isomorphism as $k$-algebras.

(3) $f^{T}: R \longrightarrow\left(R^{S T}\right)^{T}$ defined by $f^{T}(r)=f(r)^{T}=\left(r^{S T}\right)^{T}$ for all $r \in R$ is a maximality preserving isomorphism as $k$-algebras.

Furthermore, we can consider some maximality preserving maps on $M C_{n}(k)$ as following theorem:

Theorem 2.8. Suppose $R$ is an algebra in $M C_{n}(k)$. Then the following properties hold:

(1) For an invertible matrix $P \in M_{n}(k)$, the map $\psi: R \longrightarrow\left(P R P^{-1}\right)^{S T}$ defined by $\psi(r)=\left(P r P^{-1}\right)^{S T}$ for all $r \in R$ is a maximality preserving isomorphism as $k$-algebras.

(2) For an invertible matrix $P \in M_{n}(k)$, the $\operatorname{map} \phi: R \longrightarrow P R^{S T} P^{-1}$ defined by $\phi(r)=P r^{S T} P^{-1}$ for all $r \in R$ is a maximality preserving isomorphism as $k$-algebras.

Proof. (1) Since $P R P^{-1}$ is an algebra in $M C_{n}(k),\left(P R P^{-1}\right)^{S T}$ is an algebra in $M C_{n}(k)$ by Theorem 2.4. Thus, the map $\psi$ is a well defined maximality preserving map. Moreover, we can show that $\psi$ is an isomorphism as $k$-algebras by using Theorem 2.2 and Theorem 2.3. Also, (2) can be proved by similar way.

We can easily prove the following properties.

Lemma 2.9. Suppose $\left(R, m_{R}, k\right)$ is an algebra in $M C_{n}(k)$. Then, the set $\Delta=$ $\left\{r_{1}, r_{2}, \ldots, r_{t}\right\}$ is a basis of $m_{R}$ if and only if the set $\Delta^{S T}=\left\{r_{1}^{S T}, r_{2}^{S T}, \ldots, r_{t}^{S T}\right\}$ is a basis of $m_{R}^{S T}$.

By the straightforward calculations using Lemma 2.9, the next properties hold. We will let $m_{R^{S T}}$ be the maximal ideal of $R^{S T}$ and $i\left(m_{R^{S T}}\right)$ be the index of nilpotency of maximal ideal $m_{R^{S T}}$.

Theorem 2.10. Suppose $\left(R, m_{R}, k\right)$ is an algebra in $M C_{n}(k)$. Then, we have the following properties:

(1) $\operatorname{dim}\left(m_{R}\right)=\operatorname{dim}\left(m_{R^{S T}}\right)$,

(2) $\operatorname{dim}(\operatorname{soc}(R))=\operatorname{dim}\left(\operatorname{soc}\left(R^{S T}\right)\right)$,

(3) $i\left(m_{R}\right)=i\left(m_{R^{S T}}\right)$.

\section{Algebras in $M C_{n}(k)$ with $r^{S T}=r$}

Let $R$ be an algebra as in Theorem 1.1 and define a set $S$ as follows:

$$
S=\left\{r \in R \mid r^{S T}=r\right\} .
$$


Then, obviously the maximal ideal of $S$ consists of the following form of matrices:

$$
r=\left(\begin{array}{lll}
O_{\ell \times \ell} & O_{\ell \times p} & O_{\ell \times \ell} \\
A(r)_{p \times \ell} & B(r)_{p \times p} & O_{p \times \ell} \\
C(r)_{\ell \times \ell} & A(r)_{\ell \times p}^{S T} & O_{\ell \times \ell}
\end{array}\right)
$$

where $C(r)_{\ell \times \ell}^{S T}=C(r)_{\ell \times \ell}$ and $B(r)_{p \times p}^{S T}=B(r)_{p \times p}$.

Moreover, we have the following Remark 3.1:

Remark 3.1. Suppose $R$ is an algebra in $M C_{n}(k)$. Then, the algebra $S=\{r \in$ $\left.R \mid r^{S T}=r\right\}$ is a commutative subalgebra of $R$ but we can not guarantee that $S$ is an algebra in $M C_{n}(k)$ since $S$ doesn't contain all the elements of the following form

$$
r=\left(\begin{array}{lll}
O_{\ell \times \ell} & O & O \\
O & O_{p \times p} & O \\
C(r) & O & O_{\ell \times \ell}
\end{array}\right)
$$

which should be in $\operatorname{soc}(S)$. Thus, we can not say $S$ is an algebra in $M C_{n}(k)$.

Example 3.2. Let

$$
R=\left\{\left(\begin{array}{ccc}
a & 0 & 0 \\
b & a & 0 \\
c & 0 & a
\end{array}\right) \mid a, b, c \in k\right\} .
$$

Then, $S=\left\{r \in R \mid r^{S T}=r\right\}=k\left[E_{31}\right] \notin M C_{3}(k)$.

But, if $\operatorname{dim}_{k}(\operatorname{soc}(R))=1$, then the algebra $S=\left\{r \in R \mid r^{S T}=r\right\}$ can be in $M C_{n}(k)$ as the following Theorem 3.3.

Theorem 3.3. Suppose $\left(R, m_{R}, k\right)$ is an algebra in $M C_{n}(k)$ with $i\left(m_{R}\right)=3$ as in Remark 1.2 and $\operatorname{dim}(\operatorname{soc}(R))=1$. Let the maximal ideal of $S$ be $m_{S}=$ $\left\{r_{i} \mid i=1,2, \ldots, t\right\}$. If the rank of the matrix $H=\left(A\left(r_{1}\right), \ldots, A\left(r_{t}\right)\right)$ is $p$, then $S$ is an algebra in $M C_{n}(k)$.

Proof. Obviously, the algebra $S$ is a commutative algebra. Now, let $L \in M_{n}(k)$ be a matrix in the centralizer of $S$. Then, $L r_{i}=r_{i} L$ for all $r_{i} \in m_{S}$. Let $L$ and $r_{i}$ be as following block matrices:

$$
L=\left(\begin{array}{ccc}
T_{1} & T_{2} & T_{3} \\
T_{4} & T_{5} & T_{6} \\
T_{7} & T_{8} & T_{9}
\end{array}\right), \quad r_{i}=\left(\begin{array}{ccc}
O_{1 \times 1} & O & O \\
A\left(r_{i}\right) & O_{p \times p} & O \\
C\left(r_{i}\right) & A\left(r_{i}\right)^{S T} & O_{1 \times 1}
\end{array}\right),
$$

where $T_{1} \in k, T_{5} \in M_{p \times p}(k), T_{9} \in k, i=1, \ldots, t$. Then, from the relation, $L r_{i}=r_{i} L$, the following equations hold:
(1) $T_{2} A\left(r_{i}\right)+T_{3} C\left(r_{i}\right)=0$
(2) $T_{3} A\left(r_{i}\right)^{S T}=O_{1 \times q}$,
(3) $T_{5} A\left(r_{i}\right)+T_{6} C\left(r_{i}\right)=A\left(r_{i}\right) T_{1}$,
(4) $T_{6} A\left(r_{i}\right)^{S T}=A\left(r_{i}\right) T_{2}$,
(5) $A\left(r_{i}\right) T_{3}=O_{q \times 1}$, 
(6) $T_{8} A\left(r_{i}\right)+T_{9} C\left(r_{i}\right)=C\left(r_{i}\right) T_{1}+A\left(r_{i}\right)^{S T} T_{4}$,

(7) $T_{9} A\left(r_{i}\right)^{S T}=C\left(r_{i}\right) T_{2}+A\left(r_{i}\right)^{S T} T_{5}$,

(8) $C\left(r_{i}\right) T_{3}+A\left(r_{i}\right)^{S T} T_{6}=0$.

In the equation (1), if we let $C\left(r_{i}\right)=0 \in k$, then we have $T_{2} A\left(r_{i}\right)=0$ for all $i$. Thus, we obtain $T_{2}=O_{1 \times p}$ since the rank of the matrix $H=\left(A\left(r_{1}\right), \ldots, A\left(r_{t}\right)\right)$ is $p$.

Again, from the equation (1), $T_{3} C\left(r_{i}\right)=0$ implies $T_{3}=0$ by letting $C\left(r_{i}\right) \neq$ $0 \in k$. Thus, $A\left(r_{i}\right)^{S T} T_{6}=0$ for all $i$ in the equation (8) and so $T_{6}^{S T} A\left(r_{i}\right)=$ $\left(A\left(r_{i}\right)^{S T} T_{6}\right)^{S T}=0$. Thus, $T_{6}^{S T}=O_{1 \times p}$ and so $T_{6}=O_{p \times 1}$.

Now, by letting $A\left(r_{i}\right)=O_{p \times 1}$, and $C\left(r_{i}\right) \neq 0 \in k$ in the equation (6), we have $T_{1}=T_{9}=a \in k$.

Finally, in the equation (3), by letting $C\left(r_{i}\right)=0$, we have $T_{5} A\left(r_{i}\right)=$ $A\left(r_{i}\right) T_{1}=a A\left(r_{i}\right)$. This implies, if we let $I_{p}$ be the $p \times p$ identity matrix, we obtain $\left(T_{5}-a I_{p}\right) A\left(r_{i}\right)=O_{p \times 1}$ for all $i$. Thus, $T_{5}-a I_{p}=O_{p \times p}$ and so $T_{5}=a I_{p}$. Therefore, the matrix $L$ is of the form

$$
L=\left(\begin{array}{lll}
a & O & O \\
T_{4} & a I_{p} & O \\
T_{7} & T_{8} & a
\end{array}\right)
$$

In the equation (6), by letting $C\left(r_{i}\right)=0$, we obtain

$$
T_{8} A\left(r_{i}\right)=A\left(r_{i}\right)^{S T} T_{4}=\left(A\left(r_{i}\right)^{S T} T_{4}\right)^{S T}=T_{4}^{S T} A\left(r_{i}\right)
$$

since $A\left(r_{i}\right)^{S T} T_{4} \in k$. Thus, we have $\left(T_{8}-T_{4}^{S T}\right) A\left(r_{i}\right)=0$ and so $T_{8}=T_{4}^{S T}$. Therefore, $L$ is of the following form:

$$
L=\left(\begin{array}{ccc}
a & O & O \\
T_{4} & a I_{p} & O \\
T_{7} & T_{4}^{S T} & a
\end{array}\right) \in S
$$

and we can conclude that the algebra $S$ is in $M C_{n}(k)$.

Theorem 3.4. Suppose $\left(R, m_{R}, k\right)$ is an algebra in $M C_{n}(k)$ with $i\left(m_{R}\right)=3$. If $S$ is an algebra in $M C_{n}(k)$, then $\operatorname{dim}(\operatorname{soc}(R))=1$.

Proof. Suppose $\operatorname{dim}_{k}(\operatorname{soc}(R)) \neq 1$. Then, $\operatorname{dim}_{k}(\operatorname{soc}(R))=q^{2}$ for some positive integer $q$. From the condition $r=r^{S T}$, all the matrices in $\operatorname{soc}(R)$ can't be contained in $\operatorname{soc}(S)$, which contradicts to the fact in Theorem 1.1. Thus we have the result.

Corollary 3.5. Suppose $\left(R, m_{R}, k\right)$ is an algebra in $M C_{n}(k)$ with $i\left(m_{R}\right)=3$. Furthermore, assume $r^{S T}=r$ for all $r \in R$. Then $R=k\left[E_{21}+E_{n(n-1)}\right.$, $\left.\ldots, E_{(n-1) 1}+E_{n 2}, E_{n 1}\right]$.

Proof. Since $\operatorname{dim}_{k}(\operatorname{soc}(R))=1$ by Theorem $3.4, \ell=q=1$ in Theorem 1.1. Also, we may assume $R$ is the algebra of the form in Theorem 1.3. Since the rank of the matrix $H=\left(A\left(r_{1}\right), \ldots, A\left(r_{t}\right)\right)$ is $p$ and $A\left(r_{1}\right), \ldots, A\left(r_{t}\right)$ are linearly independent, we should have $p=t=n-2$ and $R=k\left[E_{21}+\right.$ $\left.E_{n(n-1)}, \ldots, E_{(n-1) 1}+E_{n 2}, E_{n 1}\right]$. 
Example 3.6. Suppose $\left(R, m_{R}, k\right)$ is an algebra in $M C_{4}(k)$ with $i\left(m_{R}\right)=3$ and $r^{S T}=r$ for all $r \in R$. Then, by Theorem 3.4 and Corollary 3.5, we obtain $\operatorname{dim}(\operatorname{soc}(R))=1$ and $R=k\left[E_{21}+E_{43}, E_{31}+E_{42}, E_{41}\right]$.

Corollary 3.7. Suppose $\left(R, m_{R}, k\right)$ is an algebra in $M C_{n}(k)$ with $i\left(m_{R}\right)=3$ and $r^{S T}=r$ for all $r \in R$. Then $\operatorname{dim}_{k}(R)=n$.

Proof. Since $R=k\left[E_{21}+E_{n(n-1)}, \ldots, E_{(n-1) 1}+E_{n 2}, E_{n 1}\right]$ by Corollary 3.5, we obtain $\operatorname{dim}_{k}(R)=1+(n-2)+1=n$.

By Corollary 3.7, we can always construct an algebra $R \in M C_{n}(k)$ with $i\left(m_{R}\right)=3$ and $\operatorname{dim}(R)=n$. Furthermore, the next corollary holds.

Corollary 3.8. Suppose $\left(R, m_{R}, k\right)$ is an algebra in $M C_{n}(k)$ such that $r^{S T}=r$ for all $r \in R$. Then, $i\left(m_{R}\right) \geq 3$ for all $n \geq 3$.

Proof. Suppose $i\left(m_{R}\right)=2$. Then, $m_{R}=\operatorname{soc}(R)$. Since $r^{S T}=r$ for all $r \in R$, $C(r)_{q \times \ell}$ should be square matrix in Theorem 1.1. Hence if $r \in \operatorname{soc}(R)$, then $C(r)_{q \times q}=C(r)_{q \times q}^{S T}$ and so $\operatorname{soc}(R)$ can't contain all the matrices of the form in Theorem 1.1 which is impossible. Therefore, $i\left(m_{R}\right) \geq 3$ for all $n \geq 3$.

\section{Algebras in $M C_{n}(k)$ with $i\left(m_{R}\right)=n=\operatorname{dim}(R)$}

In this section, we will provide a method to construct algebras $\left(R, m_{R}, k\right)$ in $M C_{n}(k)$ that $i\left(m_{R}\right)=n=\operatorname{dim}(R)$. Specially, $r=r^{S T}$ for all $r \in m_{R}$.

Let $\left(B, m_{B}, k\right)$ be a finite dimensional commutative local $k$-algebra with identity and $N$ a finitely generated faithful $B$-module. Suppose

$$
B \cong \operatorname{Hom}_{B}(N, N)
$$

via the regular representation. Define an algebra $R$ as follows:

$$
R=B\left[X_{1}, X_{2}, \ldots, X_{n-2}\right] / I,
$$

where $I$ is the following ideal:

$I=\left(m_{B} X_{1}, \ldots, m_{B} X_{n-2}, X_{1}^{2}-X_{2}, X_{1}^{3}-X_{3}, \ldots, X_{1}^{n-2}-X_{n-2}, X_{1}^{n-1}-z, X_{1}^{n}\right)$.

Here, $z$ is a nonzero element in $\operatorname{soc}(B)$ with $\operatorname{dim}_{k}(N z)=1$.

Theorem 4.1. Suppose $R$ is an algebra as in the above statements. If we let $M=N \oplus\left(\oplus_{i=1}^{n-2} N z\right)$, then the k-algebra $R$ is isomorphic to $\operatorname{Hom}_{R}(M, M)$ via the regular representation. In other words, $R$ is in $M C_{n}(k)$, where $n=$ $\operatorname{dim}_{k}(M)$. 
Proof. Obviously $M=N \oplus\left(\oplus_{i=1}^{n-2} N z\right)$ is a $B\left[X_{1}, X_{2}, \ldots, X_{n-2}\right]$-module via the following operations:

$$
\begin{array}{ccc}
\left(u, u_{1} z, \ldots, u_{n-2} z\right) b & = & \left(b u, u_{1} z b, \ldots, u_{n-2} z b\right) \\
\left(u, u_{1} z, \ldots, u_{n-2} z\right) X_{1} & = & \left(u_{1} z, u_{2} z, \ldots, u_{n-2} z, u z\right) \\
\left(u, u_{1} z, \ldots, u_{n-2} z\right) X_{2} & = & \left(u_{2} z, u_{3} z, \ldots, u z, u_{1} z^{2}\right) \\
\left(u, u_{1} z, \ldots, u_{n-2} z\right) X_{3} & = & \left(u_{3} z, u_{4} z, \ldots, u_{1} z^{2}, u_{2} z^{2}\right) \\
\vdots & \vdots & \vdots \\
\left(u, u_{1} z, \ldots, u_{n-2} z\right) X_{n-2} & = & \left(u_{n-2} z, u z, \ldots, u_{n-3} z^{2}\right),
\end{array}
$$

where $b \in B$ and $u, u_{j} \in N$ for $j=1,2, \ldots, n-2$.

Moreover, if we let $x_{j}$ is the image of $X_{j}$ in $R$ for each $j=1,2, \ldots, n-2$, then $M$ is an $R$-module via the following operations:

$$
\begin{array}{ccc}
\left(u, u_{1} z, \ldots, u_{n-2} z\right) b & = & \left(b u, u_{1} z b, \ldots, u_{n-2} z b\right) \\
\left(u, u_{1} z, \ldots, u_{n-2} z\right) x_{1} & = & \left(u_{1} z, u_{2} z, \ldots, u_{n-2} z, u z\right) \\
\left(u, u_{1} z, \ldots, u_{n-2} z\right) x_{2} & = & \left(u_{2} z, u_{3} z, \ldots, u z, u_{1} z^{2}\right) \\
\left(u, u_{1} z, \ldots, u_{n-2} z\right) x_{3} & = & \left(u_{3} z, u_{4} z, \ldots, u_{1} z^{2}, u_{2} z^{2}\right) \\
\vdots & \vdots & \vdots \\
\left(u, u_{1} z, \ldots, u_{n-2} z\right) x_{n-2} & = & \left(u_{n-2} z, u z, \ldots, u_{n-3} z^{2}\right),
\end{array}
$$

where $b \in B$ and $u, u_{j} \in N$ for $j=1,2, \ldots, n-2$.

Furthermore, by straightforward calculations, we obtain

$$
I \subseteq \operatorname{Ann}_{B\left[X_{1}, X_{2}, \ldots, X_{n-2}\right]}(M) .
$$

To show the faithfulness of $M$, let

$$
(u, 0, \ldots, 0)\left(b+\alpha_{1} x_{1}+\alpha_{2} x_{2}+\cdots+\alpha_{n-2} x_{n-2}\right)=(0,0, \ldots, 0)
$$

for $u \in N$ and $\alpha_{j} \in k$ for all $j$. Then we have

$$
\left(u b, u \alpha_{n-2} z, \ldots, u \alpha_{1} z\right)=(0,0, \ldots, 0) .
$$

Since $N$ is a faithful $B$-module by assumption, we have

$$
b=0, \quad \alpha_{j}=0, \quad j=1,2, \ldots, n-2
$$

which implies $M$ is a finitely generated faithful $R$-module.

Now, let $f \in \operatorname{Hom}_{R}(M, M)$. Define $\phi_{1}: N \rightarrow M$ and $\phi_{2}: M \rightarrow N$ by

$$
\phi_{1}(u)=(u, 0, \ldots, 0), \quad \phi_{2}\left(u, u_{1} z, u_{2} z, \ldots, u_{n-2} z\right)=u .
$$

Then, obviously $\phi_{1}$ and $\phi_{2}$ are $B$-module homomorphisms. Moreover, the map $\phi: N \rightarrow N$ defined by

$$
\phi=\phi_{2} f \phi_{1}
$$

is a $B$-module homomorphism. Since $B \cong \operatorname{Hom}_{B}(N, N)$ via the regular representation, $\phi=\mu_{a}$ for some $a \in B$, where $\mu_{a}: N \rightarrow N$ is the natural homomorphism defined by $\mu_{a}(u)=u a$ for all $u \in N$. Thus, we have

$$
\phi_{2}(f(u, 0, \ldots, 0))=\phi_{2} f \phi_{1}(u)=\phi(u)=\mu_{a}(u)=u a .
$$


By the definition of $\phi_{2}$, there exist $n-2$ number of $B$-module homomorphisms $\psi_{j} ; N \rightarrow N z$ for $j=1,2, \ldots, n-2$ such that

$$
f(u, 0, \ldots, 0)=\left(u a, \psi_{1}(u), \psi_{2}(u), \ldots, \psi_{n-2}(u)\right) .
$$

Since $\operatorname{dim}_{k}(N z)=1$, there exists an element $v \in N$ such that $\{v z\}$ is a $k$-vector space basis of $N z$. Thus, there exist $c_{1}, c_{2}, \ldots, c_{n-2} \in k$ such that

$$
\psi_{j}(v)=c_{j} v z, \quad j=1,2, \ldots, n-2 .
$$

Then, $a+c_{n-2} x_{1}+c_{n-2} x_{2}+\cdots+c_{1} x_{n-2} \in R$ and we want to show

$$
f=\mu_{a+c_{n-2} x_{1}+c_{n-3} x_{2}+\cdots+c_{1} x_{n-2}} .
$$

Since $v z$ generates $N z$, we can write

$$
u_{j} z=s_{j} v z, \quad j=1,2, \ldots, n-2
$$

for some $s_{j} \in k, j=1,2, \ldots, n-2$. Thus,

$$
\left(u, u_{1} z, u_{2} z, \ldots, u_{n-2} z\right)=\left(u, s_{1} v z, s_{2} v z, \ldots, s_{n-2} v z\right)
$$

and so we want to show that

$$
\begin{aligned}
& f\left(u, s_{1} v z, \ldots, s_{n-2} v z\right) \\
= & \mu_{a+c_{n-2} x_{1}+c_{n-3} x_{2}+\cdots+c_{1} x_{n-2}}\left(u, s_{1} v z, s_{2} v z, \ldots, s_{n-2} v z\right) .
\end{aligned}
$$

Briefly, let

$$
\begin{aligned}
r & =a+c_{n-2} x_{1}+c_{n-3} x_{2}+\cdots+c_{1} x_{n-2}, \\
w & =\left(u, s_{1} v z, \ldots, s_{n-2} v z\right) .
\end{aligned}
$$

Then, $\mu_{r}(w)$ is as follows:

$$
\begin{aligned}
\mu_{r}(w)= & \left(u, s_{1} v z, \ldots, s_{n-2} v z\right)\left(a+c_{n-2} x_{1}+c_{n-3} x_{2}+\cdots+c_{1} x_{n-2}\right) \\
= & \left(u a, s_{1} v z a, \ldots, s_{n-2} v z a\right)+\left(c_{n-2} s_{1} v z, c_{n-2} s_{2} v z, \ldots, c_{n-2} u z\right) \\
& +\cdots+\left(c_{1} s_{n-2} v z, c_{1} u z, \ldots, c_{1} s_{n-3} v z^{2}\right) .
\end{aligned}
$$

But, for each $i$ and $j$, we have

$$
\psi_{i}\left(s_{j} v\right)=s_{j} \psi_{i}(v)=s_{j} c_{i} v z .
$$

Thus, we have the following identities:

$$
\begin{aligned}
f\left(0, s_{1} v z, \ldots, s_{n-2} v z\right)= & f\left(s_{n-2} v, 0, \ldots, 0\right) x_{1}+\left(s_{n-3} v, 0, \ldots, 0\right) x_{2} \\
& \left.+\cdots+\left(s_{1} v, 0, \ldots, 0\right) x_{n-2}\right) \\
= & \left(s_{n-2} v a, \psi_{1}\left(s_{n-2} v\right), \ldots, \psi_{n-2}\left(s_{n-2} v\right)\right) x_{1} \\
& +\left(s_{n-3} v a, \psi_{1}\left(s_{n-3} v\right), \ldots, \psi_{n-2}\left(s_{n-3} v\right)\right) x_{2} \\
& +\cdots+\left(s_{1} v a, \psi_{1}\left(s_{1} v\right), \ldots, \psi_{n-2}\left(s_{1} v\right)\right) x_{n-2} \\
= & \left(\psi_{1}\left(s_{n-2} v\right), \psi_{2}\left(s_{n-2} v\right), \ldots, \psi_{n-2}\left(s_{n-2} v\right), s_{n-2} v a z\right) \\
& +\left(\psi_{2}\left(s_{n-3} v\right), \psi_{3}\left(s_{n-3} v\right), \ldots, s_{n-3} v a z, \psi_{1}\left(s_{n-3} v\right) z\right) \\
& +\cdots+\left(\psi_{n-2}\left(s_{1} v\right), s_{1} v a z, \ldots, \psi_{n-3}\left(s_{1} v\right) z\right)
\end{aligned}
$$




$$
\begin{aligned}
= & \left(c_{1} s_{n-2} v z, c_{2} s_{n-2} v z, \ldots, c_{n-2} s_{n-2} v z, s_{n-2} v a z\right) \\
& +\left(c_{2} s_{n-3} v z, c_{3} s_{n-3} v z, \ldots, s_{n-3} v a z, c_{1} s_{n-3} v z^{2}\right) \\
& +\cdots+\left(c_{n-2} s_{1} v z, s_{1} v a z, \ldots, c_{n-3} s_{1} v z^{2}\right) \\
= & \left(c_{1} s_{n-2} v z+\cdots+c_{n-2} s_{1} v z, \ldots, s_{n-2} v a z\right) .
\end{aligned}
$$

Since we can rewrite $u z=s v z$ for some $s \in k$, we have the following identities:

$$
\begin{aligned}
\left(\psi_{1}(u), \psi_{2}(u), \ldots, \psi_{n-2}(u), u a z\right) & =\left(u a, \psi_{1}(u), \psi_{2}(u), \ldots, \psi_{n-2}(u)\right) x_{1} \\
& =f(u, 0, \ldots, 0) x_{1}=f\left((u, 0, \ldots, 0) x_{1}\right) \\
& =f(0,0, \ldots, 0, u z)=f(0,0, \ldots, 0, s v z) \\
& =\left(c_{1} s v z, c_{2} s v z, \ldots, c_{n-2} s v z, s v a z\right) \\
& =\left(c_{1} u z, c_{2} u z, \ldots, c_{n-2} u z, s u z\right) .
\end{aligned}
$$

This implies that

for all $j=1,2, \ldots, n-2$.

$$
\psi_{j}(u)=c_{j} u z
$$

From the above results, we have the following identity:

$$
f(u, 0, \ldots, 0)=\left(u a, \psi_{1}(u), \ldots, \psi_{n-2}(u)\right)=\left(u a, c_{1} u z, \ldots, c_{n-2} u z\right) .
$$

Therefore, we have proved

$$
f\left(u, s_{1} v z, \ldots, s_{n-2} v z\right)=\mu_{a+c_{n-2} x_{1}+\ldots+c_{1} x_{n-2}}\left(u, s_{1} v z, \ldots, s_{n-2} v z\right)
$$

for all $\left(u, s_{1} v z, \ldots, s_{n-2} v z\right) \in N \oplus\left(\oplus_{i=1}^{n-2} N z\right)$, which implies

$$
f=\mu_{a+c_{n-2} x_{1}+\cdots+c_{1} x_{n-2}} .
$$

Since $M$ is a faithful $R$-module, we can conclude $R$ is isomorphic to

$$
\operatorname{Hom}_{R}(M, M)
$$

via the regular representation and so $R$ is in $M C_{n}(k)$.

Definition 4.2. We will call the algebra $R$ of the form in Theorem 4.1 a $C_{3}$-construction.

Corollary 4.3. Let $\left(R, m_{R}, k\right)$ be an algebra as in Theorem 4.1. Then,

(1) $\operatorname{dim}_{k}(R)=\operatorname{dim}_{k}(B)+(n-2)$.

(2) $x_{1}^{j}=x_{j}$ for all $j=1,2, \ldots, n-2$.

(3) $x_{1}^{n-1}=z$.

(4) $m_{R}$ is an ideal generated by $x_{1}$.

(5) $i\left(m_{R}\right)=n$.

Remark 4.4. If we choose an algebra $B$ with $\operatorname{dim}(B)=2$ in Theorem 4.1 , then $\operatorname{dim}(R)=n$ and so we can always construct algebras $\left(R, m_{R}, k\right) \in M C_{n}(k)$ with $i\left(m_{R}\right)=n=\operatorname{dim}(R)$.

The following is an example of a $C_{3}$-construction. 
Example 4.5. Let $\left(R, m_{R}, k\right)$ be a $k$-algebra in $M C_{n}(k)$ with $m_{R}=\left(E_{21}+\right.$ $\left.E_{32}+\cdots+E_{n(n-1)}\right)$, the ideal generated by $E_{21}+E_{32}+\cdots+E_{n(n-1)}$. Then, $R$ is the algebra defined as following:

$R=\left\{\left(\begin{array}{cccccc}a & 0 & 0 & \ldots \ldots \ldots & 0 & 0 \\ a_{1} & a & 0 & \ldots \ldots \ldots & 0 & 0 \\ a_{2} & a_{1} & a & \ldots \ldots \ldots & 0 & 0 \\ a_{3} & a_{2} & a_{1} & \ldots \ldots \ldots & 0 & 0 \\ \vdots & \vdots & \vdots & \vdots & \vdots & \vdots \\ a_{n-3} & a_{n-4} & a_{n-5} & \ldots \ldots \ldots & 0 & 0 \\ a_{n-2} & a_{n-3} & a_{n-4} & \ldots \ldots \ldots & a & 0 \\ a_{n-1} & a_{n-2} & a_{n-3} & \ldots \ldots \ldots & a_{1} & a\end{array}\right) \mid a, a_{i} \in k, i=1, \ldots, n-1\right\}$

Note that $r^{S T}=r$ for all elements $r \in R$. Moreover, if we let

$$
\begin{array}{ccc}
x_{1} & = & E_{21}+E_{32}+\cdots+E_{n(n-1)} \\
x_{2}= & E_{31}+E_{42}+\cdots+E_{n(n-2)} \\
\vdots & \vdots & \vdots \\
x_{n-2}= & E_{(n-1) 1}+E_{n 2},
\end{array}
$$

then

(1) $\operatorname{dim}_{k}(R)=n$.

(2) $m_{R}$ is an ideal generated by $x_{1}$

(3) $x_{1}^{j}=x_{j}$ for all $j=1,2, \ldots, n-2$.

(4) $x_{1}^{n-1}=E_{n 1}$.

(5) $i\left(m_{R}\right)=n$.

(6) $r^{S T}=r$ for all $r \in R$.

\section{References}

[1] W. C. Brown, Two constructions of maximal commutative subalgebras of $n \times n$ matrices, Comm. Algebra 22 (1994), no. 10, 4051-4066.

[2] _ Constructing Maximal Commutative Subalgebras of Matrix Rings in Small Dimensions, Comm. Algebra 25 (1997), no. 12, 3923-3946.

[3] W. C. Brown and F. W. Call, Maximal commutative subalgebras of $n \times n$ matrices, Comm. Algebra 21 (1993), no. 12, 4439-4460.

[4] R. C. Courter, The dimension of maximal commutative subalgebras of $K_{n}$, Duke Math. J. 32 (1965), 225-232.

[5] N. Jacobson, Schur's Theorem on Commutative Matrices, Bull. Amer. Math. Soc. 50 (1944), 431-436.

[6] Thomas J. Laffey, The Minimal Dimension of Maximal Commutative Subalgebras of Full Matrix Algebras, Linear Algebra and Its Applications 71 (1985), 199-212.

[7] Y. Song, On the Maximal Commutative Subalgebras of 14 by 14 Matrices, Comm. Algebra 25 (1997), no. 12, 3823-3840.

[8] , Maximal Commutative Subalgebras of Matrix Algebras, Comm. Algebra 27 (1999), no. 4, 1649-1663.

[9] Notes on the Constructions of Maximal Commutative Subalgebra of $M_{n}(k)$, Comm. Algebra 29 (2001), no. 10, 4333-4339.

[10] D. A. Suprunenko and R. I. Tyshkevich, Commutative Matrices, Academic Press, 1968. 
Department of Mathematics

KWANGWOON UNIVERSITY

SEOUl 139-701, KoreA

E-mail address: yksong@kw.ac.kr 